\title{
Graphene radio frequency and microwave passive components for low cost wearable electronics
}

DOI:

10.1088/2053-1583/3/2/025021

\section{Document Version}

Accepted author manuscript

Link to publication record in Manchester Research Explorer

\section{Citation for published version (APA):}

Huang, X., Leng, T., Chang, K. H., Chen, J., Novoselov, K., \& Hu, Z. (2016). Graphene radio frequency and microwave passive components for low cost wearable electronics. 2 D Materials, 3(2), [025021].

https://doi.org/10.1088/2053-1583/3/2/025021

\section{Published in:}

2 D Materials

\section{Citing this paper}

Please note that where the full-text provided on Manchester Research Explorer is the Author Accepted Manuscript or Proof version this may differ from the final Published version. If citing, it is advised that you check and use the publisher's definitive version.

\section{General rights}

Copyright and moral rights for the publications made accessible in the Research Explorer are retained by the authors and/or other copyright owners and it is a condition of accessing publications that users recognise and abide by the legal requirements associated with these rights.

\section{Takedown policy}

If you believe that this document breaches copyright please refer to the University of Manchester's Takedown Procedures [http://man.ac.uk/04Y6Bo] or contact uml.scholarlycommunications@manchester.ac.uk providing relevant details, so we can investigate your claim.

\section{OPEN ACCESS}




\title{
2D Materials
}

\section{PAPER}

CrossMark

\section{Graphene radio frequency and microwave passive components for} low cost wearable electronics

PEVISED

4 April 2016

ACCEPTED FOR PUBLICATION

25 April 2016

PUBLISHED

DD MM 2016

\author{
Xianjun Huang ${ }^{1}$, Ting Leng ${ }^{1}, K_{\text {Kuo Hsin Chang }}^{2}$, Jia Cing Chen ${ }^{2}$, Kostya S Novoselov ${ }^{3}$ and Zhirun Hu \\ 1 School of Electrical and Electronic Engineering, University of Manchester, Manchester, M13 9PL, UK \\ 2 BGT Materials Limited, Photon Science Institute, University of Manchester, Manchester, M13 9PL, UK \\ School of Physics and Astronomy, University of Manchester, Manchester, M13 9PL, UK
}

Keywords: graphene, printed graphene, RF and microwave passive components, transmission lines, co-planar waveguides, resonators and antennas

\begin{abstract}
Graphene RF and microwave passive components such as coplanar waveguide transmission lines, open/short-circuited resonators and wideband antenna on paper substrate were designed, screen printed and characterized in this work. The experimental results demonstrate that the screen printed graphene passive components can be used for RF signal transmitting, processing and radiating/ receiving; revealing that graphene ink can be a low cost alternative to much more expensive metal nanoparticle inks, such as silver nanoparticle ink. The screen printed graphene is processed at low temperature so that it is compatible with heat-sensitive flexible materials like papers, PTFE

(Polytetrafluoroethylene) and textiles. The screen printed graphene passive components reported here are of high conductivity, high flexibility, light weight and low cost, making them ideal candidate for low cost wearable electronics. This work makes it prospective to manufacture RF and microwave passive components in mass production by screen printing in much lower cost to any other known techniques.
\end{abstract}

\section{Introduction}

Ubiquitous wireless wearable communications, internet of things and machine to machine (M2M) are some of the emerging technologies that will provide an enormous step forward for mankind in the next decade [1-3]. In all these fields, RF and microwave passive components are essential to provide basic functional blocks to any communication systems. For wearable applications, the constructing materials are required to be mechanically flexible and highly conductive at RF and microwave spectrum so to reduce Ohmic loss. Currently, several types of materials have been used to make flexible conductive sheets, such as silver nanoparticles/nanowires (AgNWs), conductive polymers, carbon nanotubes and etc. Among them, silver nanoparticles/nanowires are highly conductive [4]. However, to obtain low enough sheet resistance for RF and microwave applications, a relatively thick coating is needed [4,5], leading to high manufactory cost as silver is scarce and expensive [6]. Although conductive polymer has been used in flexible electronics such as sensors, solar cell, its conductivity is too low for RF and microwave signal transmission and radiation [7, 8]. Conductive polymer is also limited by chemical and thermal instability [9]. The other candidate carbon nanotubes, having typical sheet resistance above $50 \Omega$ sq due to high junction resistance between overlapped nanotubes [10,11], are simply not conductive enough to meet practical RF and microwave circuit requirements.

Graphene, the allotrope of carbon nanotube, is a very promising material for constructing RF and microwave passive components owing to its high conductivity and unique properties [12, 13]. To date, researchers have intensively explored the applications of graphene to make active devices such as transistors and diodes [14, 15]. However, the application of graphene in RF and microwave passive components has far lagged behind. This is because, in spite of graphene's high conductivity, both exfoliated and chemical vapor deposition graphene sheets have very high surface resistance, hindering their RF and microwave applications [16, 17]. Printed graphene sheet with sheet resistance of $65 \Omega / \mathrm{sq}$ has been reported for a wideband dipole antenna application [18]. Even 


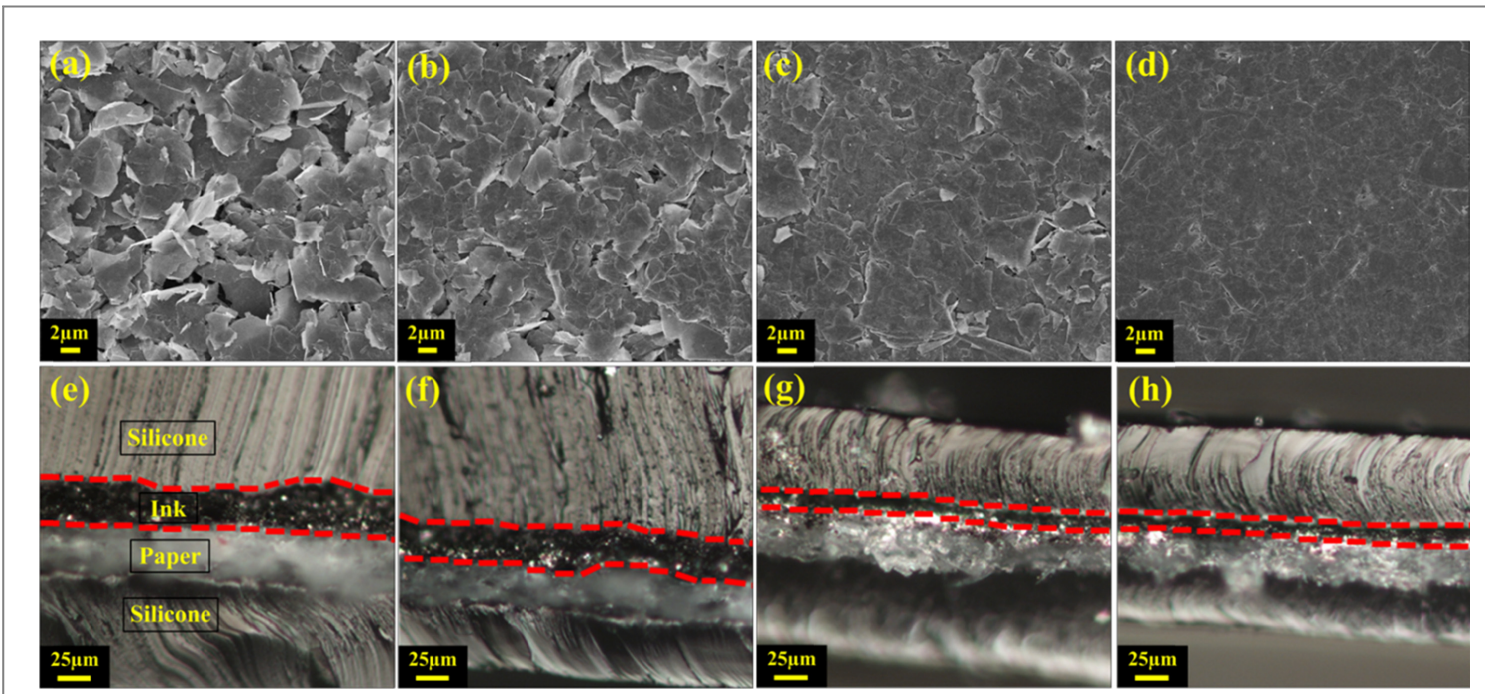

Figure 1. SEM images of top and optical microscopy images of the cross sectional views of printed graphene samples with various compression ratios. (a) Top view of un-compressed porous sample, (b) top view of sample with compression ratio $30 \%$, (c) top view of sample with compression ratio 73\%, (d) top view of sample with compression ratio $81 \%$, (e) cross sectional view of un-compressed porous sample, (f) cross sectional view of sample with compression ratio 30\%, (g) cross sectional view of sample with compression ratio $73 \%$ and $(\mathrm{h})$ cross sectional view of sample with compression ratio $81 \%$.

though good impedance matching was demonstrated, the radiation efficiency is very low due to high Ohmic loss of the printed graphene.

Recently, with reduced graphene oxide method, graphene conductive ink has been reported to have conductivity of $2.5 \times 10^{4} \mathrm{~S} \mathrm{~m}^{-1}$ [19], bringing graphene ink a step forward for practical high frequency circuit applications. Aiming for large scale industrial production, fabrication technique which combines screen printing technology and rolling compression has been developed $[20,21]$. With this technique, the printed graphene can have conductivity as high as $4.3 \times 10^{4} \mathrm{~S} \mathrm{~m}^{-1}(3.8 \Omega / \mathrm{sq})$. Moreover, the technique is characterized with low-temperature processing, which is compatible with heat-sensitive flexible substrate materials such as paper, plastics, textiles and etc. The development brings the possibility of practically printable graphene wearable electronics along with its superiority in high conductivity, mechanical flexibility, light weight and low cost [20,21].

In this work, we further apply this technique to fabricate flexible RF and microwave passive components such as coplanar waveguide transmission lines (CPW TLs), quarter wavelength open/shorted circuited resonators and wideband antenna. The performances of these components, especially under different bending cases, are experimentally examined and characterized. The results demonstrate that graphene RF and microwave passive components have desired electrical and mechanical properties for low cost wearable electronics applications.

\section{Materials preparation and methods}

Screen printing is widely used in printing industry for its advantages of high throughout and low cost. Screen printing technology together with rolling compression has been used to fabricate highly conductive graphene $\mathrm{RF}$ and microwave components. The conductive ink is commercial Gra-ink 102E from BGT Materials Ltd, which contains graphene nanoflakes, dispersants and solvents. Graphene nanoflakes were dispersed in N-Methyl-2-pyrrolidone (NMP) solvent to form a uniform $10 \mathrm{wt} \%$ graphene ink. Less than $1 \mathrm{wt} \%$ of non-ionic polymer-type surfactants was contained in the ink [20]. The surfactants improve the dispersion of graphene flakes and viscosity helping in deposition of a uniform film. The patterns for RF and microwave components are designed and fabricated on 150 mesh stainless-steel screen. Normal paper is used as a substrate and screen printing is conducted to form conductive ink pattern on the substrate. As dispersants and solvents are isolators which degrade ink conductivity, they are volatilized through a drying procedure at $100{ }^{\circ} \mathrm{C}$ for $10 \mathrm{~min}$. After the drying, the graphene nanoflakes are left on the substrate, forming a highly porous film. SEM image of top and optical microscopy image of cross sectional views of the porous nanoflake film are shown in figures 1 (a) and (e), respectively. Highly porous randomly stacked graphene nanoflakes are observable, resulting high contact resistance and unsmooth pathway for electron transport between graphene flakes. The porous film has high sheet resistance of $38 \Omega /$ sq (measured with 4 -point probe, RM3000, Jandel, UK) and its thickness is measured to be $31.6 \mu \mathrm{m}$ using digital thickness gauge (PC-485, Teclock, Japan). Its bulk conductivity can be calculated to be $8.3 \times 10^{2} \mathrm{~S} \mathrm{~m}^{-1}$, which is too low for any practical 
RF and microwave circuit applications. To improve the conductivity, a rolling compression is conducted using compression roller (SERP02, Shining Energy, Taiwan). To show the effect of rolling procedure, three more samples with compression ratios of 30\%, 73\% and $81 \%$, corresponding to thicknesses of $22.1 \mu \mathrm{m}$, $8.4 \mu \mathrm{m}$ and $6.0 \mu \mathrm{m}$ respectively, were prepared. SEM images of the top and optical microscopy images of the cross sectional views of these samples are shown in figures 1(b), (f), (c), (g), (d) and (h), respectively. For better observation and scope fitting, magnification of $20 \times$ is used for samples in figures 1(e)-(h). By comparing the front views in figures $1(a)-(d)$, it can be seen that the surface of graphene nanoflake film becomes denser and smoother with higher compression. From figures 1(e)-(h), one can see that the thickness decreases correspondingly with higher compression ratios. The sheet resistances of these compressed samples were then measured. They are $28.5 \Omega / \mathrm{sq}, 8.2 \Omega /$ sq and $3.8 \Omega / \mathrm{sq}$, corresponding to the bulk conductivities of $1.6 \times 10^{3} \mathrm{~S} \mathrm{~m}^{-1}, 1.4 \times 10^{4}$ $\mathrm{S} \mathrm{m}^{-1}$ and $4.3 \times 10^{4} \mathrm{~S} \mathrm{~m}^{-1}$ respectively. It can be seen that the conductivity of the sample in figure $1(\mathrm{~d})(81 \%$ compression ratio) is more than 50 times higher than that of uncompressed one in figure 1(a). The compression makes the printed graphene highly conductive and promising for RF and microwave circuit applications.

\section{Results and discussion}

\subsection{CPW TLs}

TLs are basic structures designed to carry signal from one point to the other and are essential for signal transmission, impedance matching network, resonators, filters and etc [22]. In order to investigate the feasibility for RF and microwave signal transmission, screen printed graphene CPW have been designed, fabricated and characterized.

CPW TLs have one conducting track and two return conductors as ground. These three conductors are all on the same plane, resulting in low-profile property for CPW based components. This advantage makes CPW TLs widely used in RF and microwave circuits. The performance of a CPW TL is mainly determined by material and structural parameters such as material losses, dielectric constant, line widths, line gaps and etc. Figure 2 shows the printed graphene CPW TL on paper substrate under different bending conditions. To measure the performance, a SMA connector is connected at each port of the line using conductive epoxy. The length of the lines is $l$ of $50 \mathrm{~mm}$, the central conductor width $w$ of $1.7 \mathrm{~mm}$ and the gaps between ground and central line $g$ of $0.5 \mathrm{~mm}$.

The scattering parameters of the CPW TL are measured using Agilent E5071B VNA. The propagation constant can be calculated using the following equation [23]

$$
\begin{gathered}
\mathrm{e}^{-\gamma l}=\frac{2 S_{21}}{1-S_{11}^{2}+S_{21}^{2} \pm \sqrt{\left(1+S_{11}^{2}-S_{21}^{2}\right)^{2}-4 S_{11}^{2}}} \\
\gamma=\alpha+\mathrm{j} \beta
\end{gathered}
$$

where $\alpha$ and $\beta$ are attenuation constant and phase constant, respectively. To eliminate the effects of impedance mismatch on analyzing the losses, absorption attenuation, which is defined as the ratio of power entered into the network over the output power of the network, is calculated [24]

$$
\text { Attenuation }=\frac{P_{\text {in }}}{P_{\text {out }}}=\frac{1-\left|S_{11}\right|^{2}}{\left|S_{21}\right|^{2}} .
$$

With measured $S$ parameters and (3), the phase constant and attenuation (per $\mathrm{mm}$ ) are calculated and displayed in figure 2(e). The two parameters, especially the phase constant, are almost linearly increasing with frequency, implying that there is little distortion in the printed graphene CPW TLs, which is desirable in practical RF and microwave applications. The relatively high attenuation is due to small thickness of the printed graphene line compared with skin depth. In this work, the thickness of the printed graphene is $7.7 \mu \mathrm{m}$, with the bulk conductivity of $4.3 \times 10^{4} \mathrm{~S} \mathrm{~m}^{-1}$. Its skin depth, at central frequency of measurement $4 \mathrm{GHz}$, is $38 \mu \mathrm{m}$, which means that the printed graphene line thickness is only $20.2 \%$ of its skin depth. To reduce attenuation in practical applications, normally conductor thickness should be 3-5 times of its skin depth. Increasing the printed graphene thickness is an effective way to obtain lower attenuation.

Moreover, the superior flexibility of the printed graphene CPW TL is experimentally verified with comparison of performances under different bending cases. As seen in figures 2(a)-(d), the CPW TL with original length of $50 \mathrm{~mm}$ is bended. The port to port distances of $40 \mathrm{~mm}, 30 \mathrm{~mm}$ and $20 \mathrm{~mm}$ were studied respectively. The transmission coefficients under these cases are given in figure 2(f). It is clearly evident that the bending does not alter the transmission coefficients much, highly desirable for wearable applications. It should be pointed out that the CPW TL in this experiment has not been optimized for $50 \Omega$ VNA measurement system. Higher transmission coefficient can be achieved with better impedance matching.

\section{2. $\mathrm{CPW}$ resonators}

Microwave resonator is one of the fundamental building blocks for RF and microwave signal processing [25]. Printed graphene CPW resonators have been designed and fabricated. The measurement results reveal that printed graphene is well suited for constructing RF resonators. Figure 3 shows the printed graphene open-circuited (OR) CPW resonator and its performance. The effective length of the resonator is $l$ of $30 \mathrm{~mm}$, the central conductor width $w$ of $1.7 \mathrm{~mm}$ and gap between central conductor and ground $g$ of $0.5 \mathrm{~mm}$. The resonator is connected to a SMA for measurement purpose. The reflection coefficient $S_{11}$ 


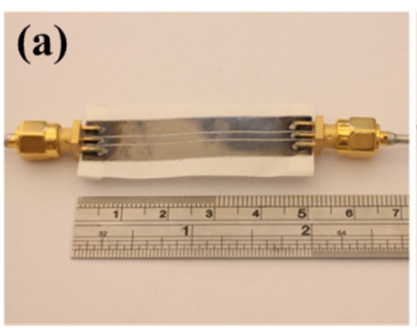

(c)

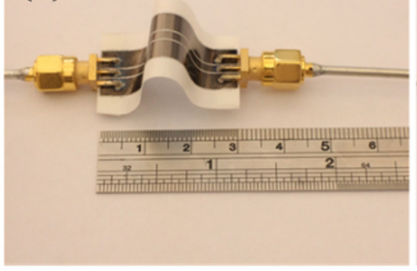

(b)

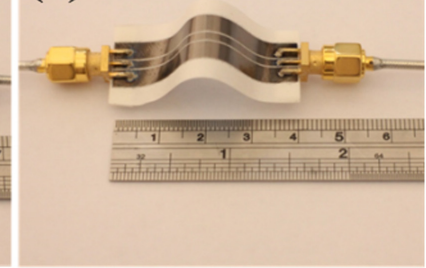

(d)

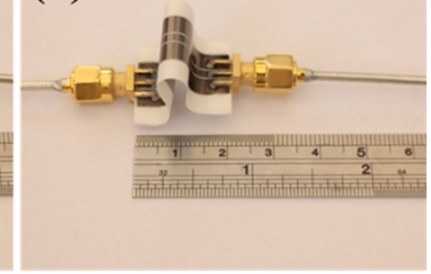

(e) 0.50
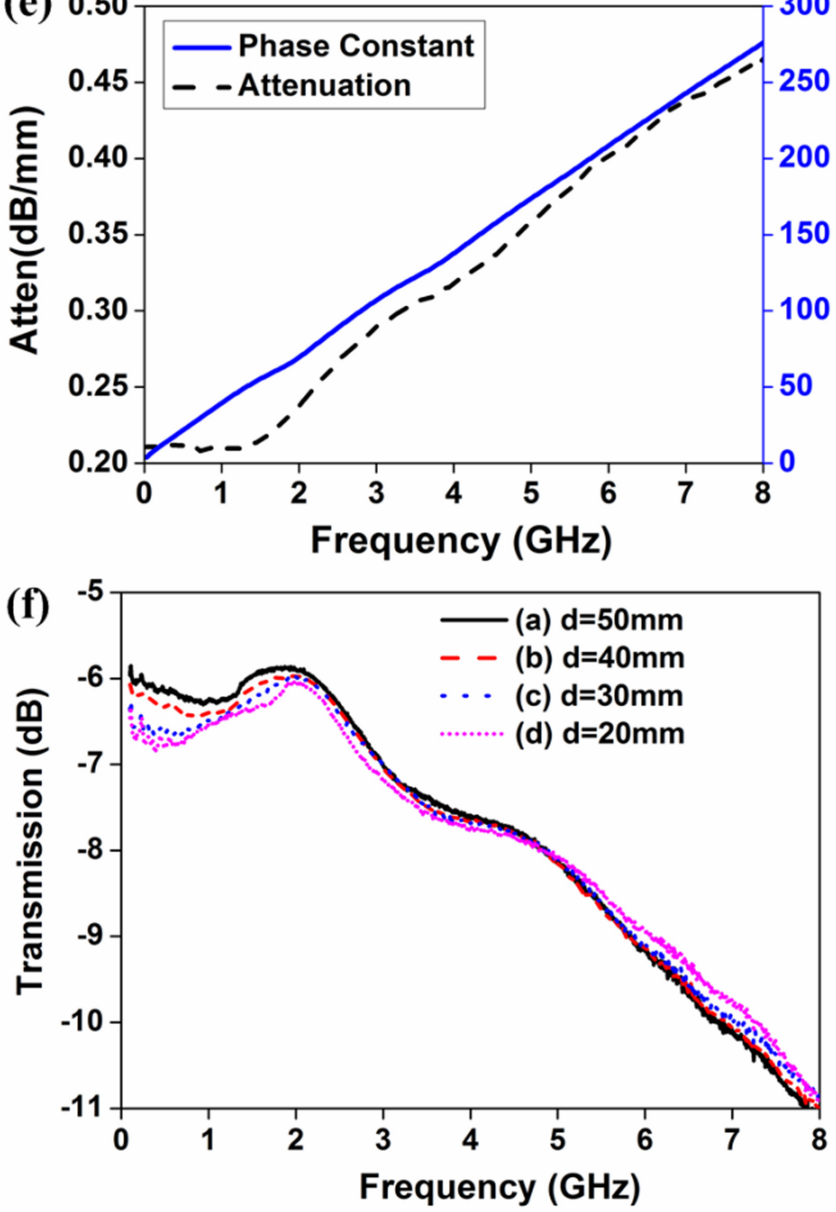

Figure 2. Coplanar waveguide transmission lines (CPW TLs) and their performance under different bending. (a) Un-bended CPW TL with original length of $50 \mathrm{~mm}$, (b) bended CPW TL, port to port distance of $40 \mathrm{~mm}$, (c) bended CPW TL, port to port distance of $30 \mathrm{~mm}$, (d) bended CPW TL, port to port distance of $20 \mathrm{~mm}$, (e) attenuation and phase constant of the un-bended CPW TL and (f) transmission coefficient of the CPW TL under different bending conditions ( $d$ is the port to port distance). 

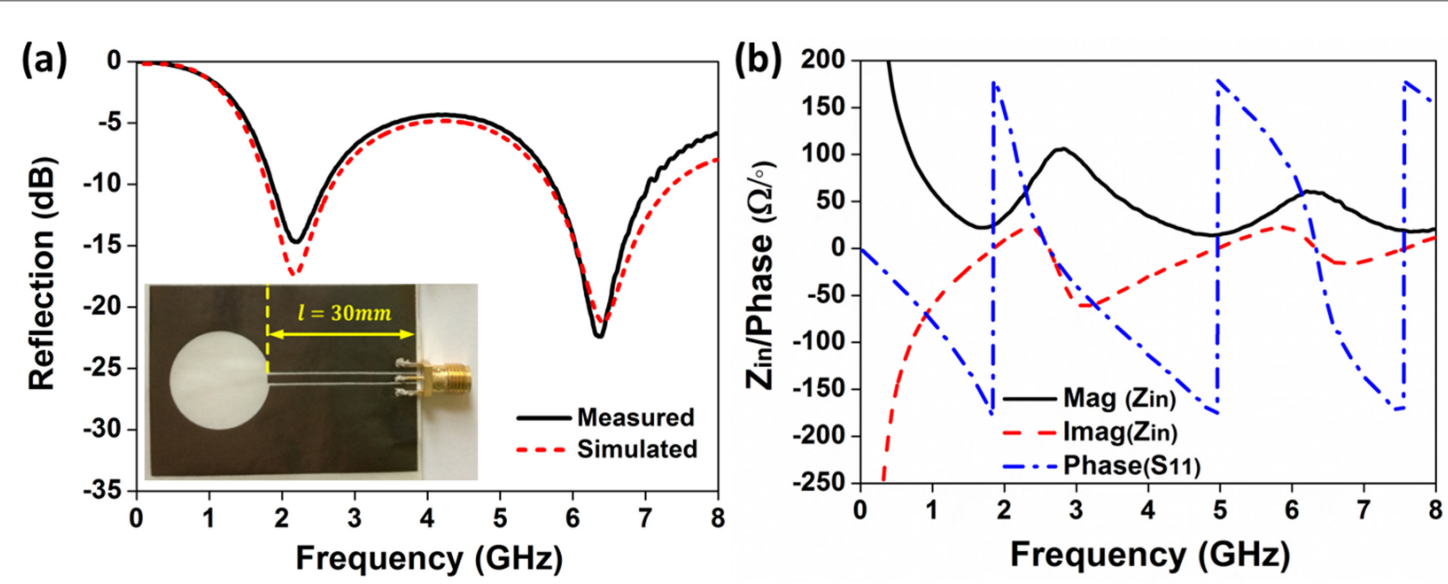

Figure 3. (a) Photo of the CPW open-circuited $\lambda / 4$ resonator printed on paper substrate and its reflection magnitude (dB) and (b) its input impedance (magnitude and phase) and reflection phase.

was measured using Agilent E5071B VNA. A numerical simulation is also carried out in CST Microwave Studio. The printed graphene in simulation is modeled as ohmic sheet with $\mathrm{Rs}=3.8 \Omega$, and the supporting paper is modeled with relative dielectric constant $\varepsilon_{\mathrm{r}}=3$ and thickness of $200 \mu \mathrm{m}$.

From $S_{11}$, the input impedance of a resonator can be calculated by

$$
Z_{\text {in }}=Z_{0} \frac{1+S_{11}}{1-S_{11}}
$$

where $Z_{0}=50 \Omega$ is the characterized impedance.

To facilitate the investigation of resonance, magnitude and phase of $S_{11}$ are separately displayed. The magnitude of $S_{11}$, both measured and simulated are shown in figure 3(a) and the phase is presented in figure 3(b) together with input impedance. The measurement and simulation result in figure 3(a) prove good match. As it can be seen in figure 3(b), as frequency approaches to zero, the phase of $S_{11}$ is zero, and input impedance reaches almost infinite because the circuit is opened. At around $1.9 \mathrm{GHz}$, the phase becomes $180^{\circ}$, the imaginary part of input impedance becomes 0 and the magnitude of input impedance reaches minimum for this open-circuited $\lambda / 4$ resonator. All these indicate that the fundamental resonance occurs at this frequency point.

The quality factor $Q$ of the open-circuited $\lambda / 4$ resonator is only about 3. The relatively low $Q$ value is mainly caused by the small thickness of the printed graphene layer ( $14 \%$ of skin depth at $1.9 \mathrm{GHz}$ ). Much higher $Q$ values can be expected if the thickness of printed graphene increases to 3-5 times of the skin depth. As a proof of concept, however, the relatively low $Q$ value reported here should not distract the potential of printed graphene for low cost and wearable RF and microwave electronics applications.

To demonstrate the flexibility, a short-circuited $\lambda /$ 4 resonator with physical length $l$ of $60 \mathrm{~mm}$ has been measured under different bending cases, as shown in figure 4. The resonator is un-bended in figure 4(a), whereas under different bending conditions in figures 4(b)-(c). The resonator in figure 4(d) has not just been bended but also twisted. The reflection coefficients for these four cases are displayed in figure 4(e). As it can be seen, the reflection coefficients of the resonator changes little, proving the resonator's superior flexibility. Also, minor difference on reflection minima intensities of bent and unbent samples is observed. This can be explained by the minor coupling between different segments in bent ones, while unbent straight device does not have this coupling.

With successful demonstration of printed graphene open/short-circuited resonators, more complex passive components used for RF and microwave signal processing, such as filters and couplers, can be constructed.

\subsection{Wideband antenna}

Wideband antennas are used for many applications such as high speed data link, microwave imaging, precise geolocation and etc [26-30]. A lightweight and flexible printed graphene wideband microwave antenna has been designed and fabricated, as shown in figure $5(\mathrm{~d})$. This is a typical CPW-fed triangle slot antenna [31]. The graphene is printed on a normal paper as conductive radiation material and the CPW port is connected to a SMA using conductive epoxy for measurement purpose.

The reflection coefficient of the antenna was measured using Agilent E5071B VNA and shown in figure 5(e) together with measured antenna gain which was obtained using three-antenna method. It can be seen that the $-10 \mathrm{~dB}$ bandwidth of the antenna is from 3.75 to $12.88 \mathrm{GHz}$, i.e., $110 \%$ fractional bandwidth. This frequency band covers many important commercial applications such as wireless communications, navigation, satellite communications and etc [32-34]. It can also be seen that a reasonably good gain within the operation bandwidth has been obtained. The maximum gain is $1.9 \mathrm{dBi}$ at $10.06 \mathrm{GHz}$. To further verify 

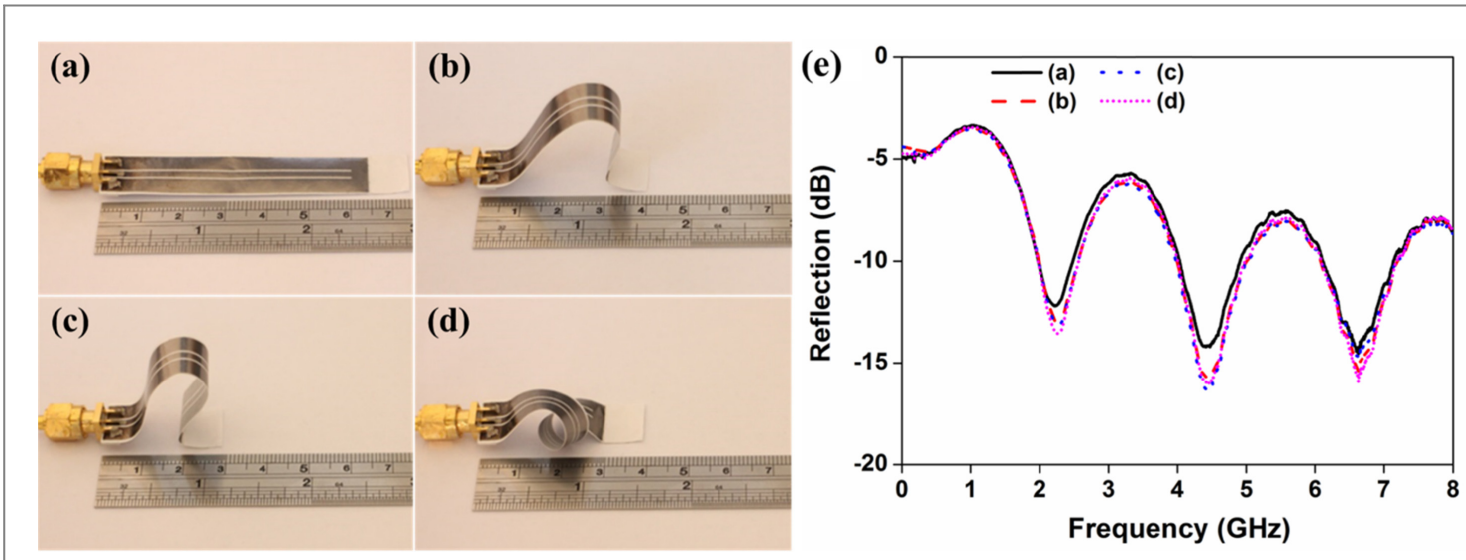

Figure 4. Flexibility of printed graphene short-circuited $\lambda / 4$ resonator. (a) Un-bended, (b) bended, (c) further bended, (d) bended and twisted and (e) reflection coefficients under different bending cases.

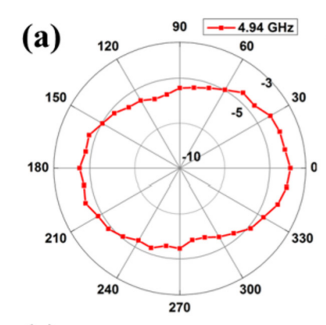

(c)

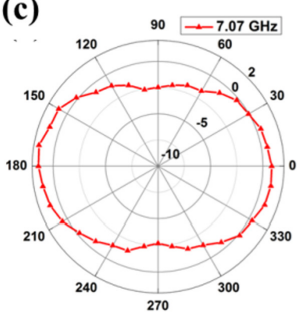

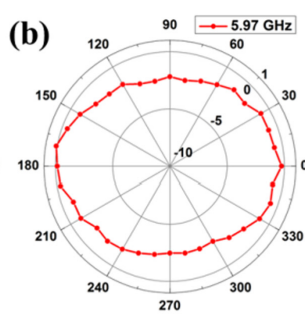

(d)

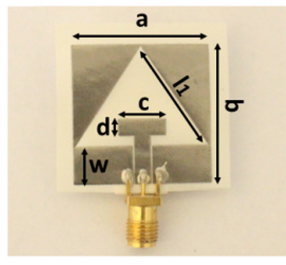

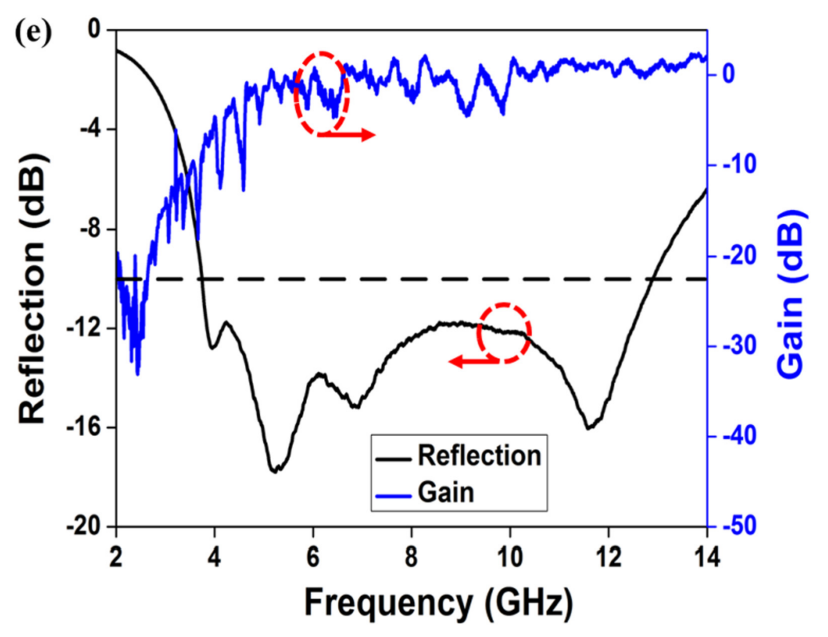

Figure 5. Printed graphene wideband antenna and its performances. (a) Radiation pattern at $4.94 \mathrm{GHz}$, (b) radiation pattern at $5.97 \mathrm{GHz},(\mathrm{c})$ radiation pattern at $7.07 \mathrm{GHz}$, (d) photo of the antenna and the dimensions are $a=b=26 \mathrm{~mm}, l_{1}=22.65, c=8.5 \mathrm{~mm}$, $d=3 \mathrm{~mm}, w=\mathrm{mm}$, the CPW line has central electron width $3 \mathrm{~mm}$ and line gap $0.35 \mathrm{~mm}$, (e) measured gain and reflection coefficients.

the radiation, the $H$-plane radiation patterns of the printed graphene antenna at $4.94 \mathrm{GHz}, 5.97 \mathrm{GHz}$ and 7.07 GHz were measured using antenna measurement system (Antenna Measurement Studio 5.5, Diamond Engineering) and illustrated in figures 5(a), (b) and (c), respectively. The data were recorded for every $10^{\circ}$ rotation. These radiation patterns demonstrate the effective radiation of the antenna. It can be seen that the maximum radiation directions at these frequencies are all at $0^{\circ}$ direction, i.e., normal to the antenna plane. The radiation patterns at these three frequencies are very similar. Such consistent radiation patterns in wide frequency band are desirable for applications such as UWB detection and wideband wireless sensing and communications.

To verify the antenna's flexibility, we have measured the antenna performances for two bended cases as shown in figure 6. The reflection coefficient of unbended antenna is also shown in the figure for comparison. The bending was made in two mutually perpendicular directions, as seen in figures 6(a) and (b). It is clearly evident that the reflection coefficients for all the cases are very similar and the $-10 \mathrm{~dB}$ bandwidths are almost the same, demonstrating superior flexibility. Such flexibility is highly desirable in many applications, especially for wearable electronics [35-37].

\section{Conclusions}

In this work, we have fabricated graphene RF and microwave passive components by combining largescale industrial screen printing technology and rolling compression procedure. The significant improvement on printed graphene's conductivity together with its flexibility has made it feasible to construct printed graphene RF and microwave passive components. CPW TLs, quarter wavelength resonators and wideband antenna have been screen printed, measured and analyzed in depth. Very good performances were 


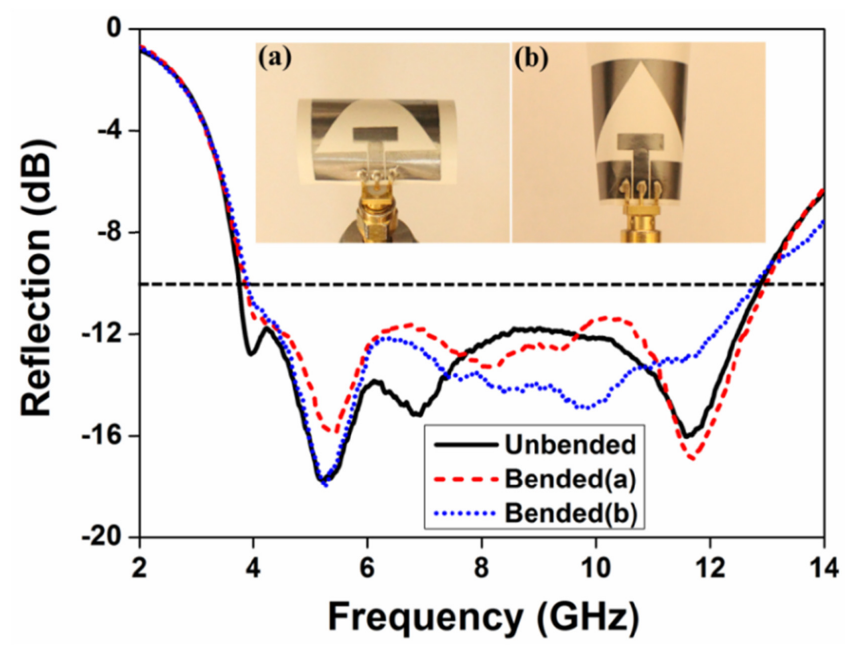

Figure 6. Performance comparison of un-bended/bended printed graphene antenna.

demonstrated experimentally, revealing that screen printed graphene RF and microwave passive components can be a promising candidate for RF and microwave circuit applications, especially where low cost, flexibility and wearability are required. This work demonstrates a step forward development for industrial scale mass production of flexible RF and microwave passive components for low cost wearable electronics applications.

\section{References}

[1] Tang S L P 2007 Recent developments in flexible wearable electronics for monitoring applications Trans. Inst. Meas. Control 29283-300

[2] Zanella A, Bui N, Castellani A, Vangelista L and Zorzi M 2014 Internet of things for smart cities IEEE Internet Things J. 1 22-32

[3] Wu G, Talwar S, Johnsson K, Himayat N and Johnson K 2011 M2M: from mobile to embedded internet IEEE Commun. Mag. 49 36-43

[4] De S, Higgins T, Lyons P, Doherty E, Nirmalraj P, Blau W, Boland J and Coleman J 2009 Silver nanowire networks as flexible, transparent, conducting films: extremely high DC to optical conductivity ratios ACS Nano 3 1767-74

[5] Song L, Myers A, Adams J and Zhu Y 2014 Stretchable and reversibly deformable radio frequency antennas based on silver nanowires ACS Appl. Mater. Interfaces 6 4248-53

[6] Zeng X, Zhang Q, Yu R and Lu C 2010 A new transparent conductor: silver nanowire film buried at the surface of a transparent polymer Adv. Mater. 22 4484-8

[7] Cochrane C, Koncar V, Lewandowski M and Dufour C 2007 Design and Development of a flexible strain sensor for textile structures based on a conductive polymer composite Sensors 7 473-92

[8] De Girolamo Del Mauro A, Diana R, Grimaldi I, Loffredo F, Morvillo P, Villani F and Minarini C 2013 Polymer solar cells with inkjet-printed doped-PEDOT: PSS anode Polym. Compos. 34 1493-9

[9] Richardson-Burns S, Hendricks J, Foster B, Povlich L Kim D and Martin D 2007 Polymerization of the conducting polymer poly(3,4-ethylenedioxythiophene) (PEDOT) around living neural cells Biomaterials 28 1539-52

[10] Hu L, Hecht D and Grüner G 2010 Carbon nanotube thin films: fabrication, properties, and applications Chem. Rev. 110 5790-844
[11] Kamyshny A and Magdassi S 2014 'Conductive nanomaterials for printed electronics Small 103515-35

[12] Lee S, Lee K, Liu C, Kulkarni G and Zhong Z 2012 Flexible and transparent all-graphene circuits for quaternary digital modulations Nat. Commun. 31018

[13] Geim A and Novoselov K 2007 The rise of graphene Nat. Mater. 6 183-91

[14] Han S, Jenkins K, Valdes Garcia A, Franklin A, Bol A and Haensch W 2011 High-frequency graphene voltage amplifier Nano Lett. 11 3690-3

[15] Yang X, Liu G, Balandin A and Mohanram K 2010 Triplemode single-transistor graphene amplifier and its applications ACS Nano $45532-8$

[16] Gomez-Diaz J and Perruisseau-Carrier J 2012 'Microwave to $\mathrm{THz}$ properties of graphene and potential antenna applications Int. Symp. on Antennas and Propagation (ISAP), 2012 pp 239-42

[17] Balanis C 2005 Antenna Theory (Hoboken, NJ: Wiley Interscience)

[18] Shin K, Hong J and Jang J 2011 'Micropatterning of graphene sheets by inkjet printing and its wideband dipole-antenna application Adv. Mater. 23 2113-8

[19] Secor E, Prabhumirashi P, Puntambekar K, Geier M and Hersam M 2013 Inkjet printing of high conductivity, flexible graphene patterns J. Phys. Chem. Lett. 4 1347-51

[20] Huang X, Leng T, Zhang X, Chen J, Chang K, Geim A, Novoselov K and Hu Z 2015 Binder-free highly conductive graphene laminate for low cost printed radio frequency applications Appl. Phys. Lett. 106203105

[21] Huang X, Leng T, Zhu M, Zhang X, Chen J, Chang K, Aqeeli M, Geim A, Novoselov K and Hu Z 2015 High flexiblw and conductive printed graphene for wireless wearable communications applications Sci. Rep. 518298

[22] Bowick C, Blyler J and Ajluni C 2008 RF Circuit Design (Amsterdam: Newnes/Elsevier)

[23] Zhang J and Hsiang T 2007 'Extraction of subterahertz transmission-line parameters of coplanar waveguides PIERS Online 3 1102-6

[24] Zhang K and Li D 2008 Electromagnetic Theory for Microwaves and Optoelectronics (Berlin: Springer)

[25] Pozar D 2005 Microwave Engineering (Hoboken, NJ: Wiley)

[26] Allen B 2007 Ultra Wideband Antennas and Propagation for Communications, Radar and Imaging (Chichester: Wiley)

[27] Song K and Xue Q 2013 Ultra-wideband ring-cavity multipleway parallel power divider IEEE Trans. Ind. Electron. 60 4737-45

[28] Mroué A, Heddebaut M, Elbahhar F, Rivenq A and Rouvaen J M 2012 Automatic radar target recognition of objects falling on railway tracks Meas. Sci. Technol. 23025401 
[29] Abbosh A, Mohammed B and Bialkowski M 2011 Planar array antenna for ultra wideband microwave imaging system Int. Conf. on Communications and Information Technology (ICCIT), 2011 (IEEE)

[30] Al Shaheen, Ahmed and Al-Rizzo H 2011 An ultra wide band antenna design for indoor geolocation applications World Appl. Sci. J. 12.8 1321-6

[31] Shameena V, Mridula S, Pradeep A, Jacob S, Lindo A and Mohanan P 2012 A compact CPW fed slot antenna for ultra wide band applications AEU-Int. J. Electron. Commun. 66 189-94

[32] Federal Communication Commission 2002 Revision of part 15 of the commission's rules regarding ultra-wideband transmission system, first note and order Washington, DC, ET-Docket 98-153
[33] Gungor V and Hancke G 2009 Industrial wireless sensor networks: challenges, design principles, and technical approaches IEEE Trans. Ind. Electron. 56 4258-65

[34] Gungor V, Sahin D, Kocak T, Ergut S, Buccella C, Cecati C and Hancke G 2011 Smart grid technologies: communication technologies and standards IEEE Trans. Ind. Inform. 7529-39

[35] Hertleer C, Rogier H, Vallozzi L and Van Langenhove L 2009 A textile antenna for off-body communication integrated into protective clothing for firefighters IEEE Trans. Antennas Propag. 57 919-25

[36] 2008 Antennas and propagation for body-centric wireless communications IEEE Antennas Propag. Mag. 50148

[37] Winterhalter C, Teverovsky J, Wilson P, Slade J, Horowitz W Tierney E and Sharma V 2005 Development of electronic textiles to support networks, communications, and medical applications in future US military protective clothing systems IEEE Trans. Inf. Technol. Biomed. 9402-6 


\section{QUERY FORM}

JOURNAL: 2D Materials

AUTHOR: X Xuang etal

TitLE: Graphene radio frequency and microwave passive components for low cost wearable electronics

ARTICLE ID: tdmaa225b

The layout of this article has not yet been finalized. Therefore this proof may contain columns that are not fully balanced/matched or overlapping text in inline equations; these issues will be resolved once the final corrections have been incorporated.

Page 1

Q1

Please specify the corresponding author and provide his/her email address.

Page 7

Q2

Please check the details for any journal references that do not have a link as they may contain some incorrect information.

Page 8

Q3

Please provide the initials for the author [Al Shaheen and Ahmed] in reference [30].

Page 8

Q4

Report reference [32] should contain author name, year, report title and institution. Please provide the missing information.

Page 8

Q5

Please provide author names or collaboration name for reference [36]. 